\title{
PROCESOS FINALES EN EL ESPANOL DE SANTO DOMINGO
}

El habla de la capa social intermedia de la ciudad de Santo Domingo acusa una serie de procesos fonológicos finales bastante importantes ${ }^{1}$; entre ellos podemos mencionar la conocida neutralización de liquida en posición final. Básicamente, el proceso consiste en transformar, con base en algunas reglas generativas, la / $r$ / subyacente en el educto fonético [1] al final de sílaba y en posición final absoluta, por ejemplo, cuerpo [kwélpo], servir [selbill, cardo [káldo] (esta última palabra sería confundida con caldo [káldo] y mar sería tomada por mal al pronunciarse de la misma manera [mál]. Si desconociéramos este dialecto, al oír estas formas por primera vez nos sería difícil determinar si estamos tratando con líquida vibrante o lateral. Si consideramos las formas siguientes, tendremos un asidero más firme para considerar [1] como proveniente de /r/ subyacente: mejol-mejores, mujel-mujeres, hablal-hablarć, peol-peores, asadol-asadcres.

Para demostrar que la representación fonológica debe ser $/ r /$ basta con señalar, por un lado, que la [l] fonética al final de palabra es, en todos los casos de modificación, una $/ r /$ intervocálica proveniente de un nivel superior de derivación; $y$, por el otro, que una regla que mude $r$ a $l$ en posición intervocálica no funcionaría por la sencilla razón de que la vibrante debe estar en posición final, según se ejemplifica más arriba. Se sabe que el cambio es de $r$ a $l$ y no viceversa, puesto que una palabra como capital se pronuncia [kapitál] sin modificación alguna de la líquida lateral.

Asimismo se registra un proceso que denominaremos formación de velar, que consiste en mudar una / $\mathrm{n} /$ fonológica en $[\mathrm{y}]$ fonética al final de palabra y ante otra nasal. Nuevamente las alternancias de varias formas nos sirven de apoyo a lo antedicho: jovey-jóvenes, virgen-vírgenes, escalón-escalones, innecesario. Este proceso, caracteristico del habla lenta y moderada, también tiene lugar en límite de palabra: tay tonto, soy hermanas, cọ razón, tạ olvidadizo, hacen casas, ey agua. En límite e interior de palabra de un habla más espontánea y rápida la nasal alveolar se asimila al punto de articulación de la consonante siguiente: compra, ímfimo, ante, chinche, camióm parado, un doctor, un queso. Permanece no obstante como velar ante vocales, semiconsonantes y en

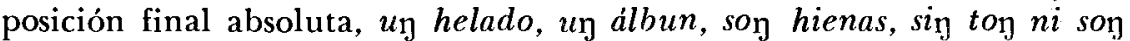

Por último, se realiza el conocido proceso de modificar la /s/ final de sílaba, y en límite de palabra, en la aspirada [h] o en el cero fonético $[\phi]$ :

1 Le estoy profundamente agradecido a Kathleen Houlihan por sus valiosas sugerencias y comentarios. Por supuesto, cualquier error de interpretación es de mi exclusiva responsabilidad. 


\begin{tabular}{ll}
$\begin{array}{l}\text { experiencia } \\
\text { resbalar }\end{array}$ & [ehperjénsja], [eperjénsja] \\
mismo & [rehßalál], [reßbalál] \\
fósforo & [míhmo], [mimo] \\
hasta & [fóhforo], [fóforo] \\
desyerbar & [áhta], [áta] \\
vez & [dehyeiß́ál], [deyelßál] \\
es mía & [béh], [be] \\
los números & [eh mía], [e mia] \\
\hline
\end{tabular}

Desde luego, la transformación no es absoluta, pues dependiendo del contexto en límite de palabra puede aparecer ya $[\mathrm{s}]$, ya $[\mathrm{h}]$ o $[\phi]$. Si la palabra siguiente se inicia con vocal, lo más probable es que $/ \mathrm{s} /$ no se modifique, sus hijos [susího(h)], pero también es muy probable encontrar las otras dos posibilidades, [lo $(h)$ e $(h)$ túdjo $(h)]$.

Estos tres procesos que hemos tratado de resumir en los párrafos anteriores han sido objeto, de una manera $u$ otra, de análisis por varios lingüistas hispanos. Quilis ${ }^{2}$ ha intentado elucidar la transformación que sufre $n$ al final de sílaba, y para tal efecto se ha valido de la definición de silaba como la menor unidad de impulso espiratorio en que se divide el habla real. Como dicho impulso consiste en una tensión seguida de una distensión muscular, Quilis observa que algunos hablantes cambian en $\mathfrak{y}$ velar a nasal alveolar $n$. El cambio radica en un proceso de "debilitamiento articulatorio que se manifiesta más señaladamente en la posición postnuclear (ibid., p. 165). Nos interesa sobremanera el proceso de debilitamiento, que Quilis menciona, y que quiere substanciar al sugerir que la "velarización" de $n$ es un primer paso hacia su pérdida y a la consiguiente nasalización de la vocal precedente. Para aceptar este tipo de razonamiento necesitamos, sin embargo, fundamentos teóricos y empíricos que nos ayuden a determinar que la modificación de $n$ a $y$ se debe a debilitamiento.

Un problema similar, aunque no idéntico, presenta Alonso ${ }^{3}$. Algunos caracteres que le son propios a una consonante en principio de sílaba pierden -dice Alonso- su "identidad en la distensión" y por ende la consonante se simplifica (ibid., p. 240). Muestra de esa simplificación en la distensión es la confusión de $r$ y $l$ que existe en algunas regiones del mundo hispánico. Dice Alonso que a pesar de eliminarse fonéticamente la oposición de estos dos segmentos, fonológicamente permanecen como distintos. Es decir que al final de sílaba se ha registrado una simplificación porque tiene lugar una neutralización ya sea de $r$ a $l$ o de $l$ a $r$, mientras que en la representación fonológica la distinción fonónica ${ }^{4}$ de estas consonantes todavía perdura, según vimos con las alternancias singular-plural de algunas formas en Santo Domingo. Alonso define, pues, el proceso de simplificación como el abandono de

2 ANTonio Quilis, "La juntura en español: un problema de fonologia", $P F E, 2$, 1963, 163.171.

G AMado Alonso, "Una ley fonológica del español", en Estudios lingü̈ísticos: te. mas españoles, Madrid, 1967, pp. 237-249.

4 De aquí en adelante emplearemos el término fonón para significar rasgo distintivo. 
algunos fonones. No obstante, habría que precisar de forma más rigurosa con referencia a qué podemos determinarlo como de naturaleza simplificadora y sólo lo podemos hacer si nos forzamos a inquirir en el nivel fonológico qué relación de fuerza tendría $/ \mathrm{r} /$ con respecto a $/ 1 /$.

Los planteamientos de Quilis se basan en el postulado que introduce Hooper ${ }^{5}$, entre otros. Para Hooper, los procesos finales de sílaba son de debilitamiento y los justifica a base de una escala consonántica de fuerza que comentamos abajo. En este ensayo intentaremos resolver dos cuestiones fundamentales: 1) establecer una escala de consonantes para sostener el proceso que ocurre en la distensión silábica como de debilitamiento y 2) demostrar que si los procesos que experimentan las consonantes al final de sílaba son de debilitamiento, entonces el español de Santo Domingo, con la neutralización de $r$ a $l$, aporta evidencias que desmienten tales premisas. Siguiendo la hipótesis de Houlihan ${ }^{6}$, diremos que la neutralización al final de sílaba puede ser de fuerza, si es que deseamos mantener una única escala consonántica independiente de la posición en la palabra e independiente de los procesos fonológicos que les son específicos a una lengua dada.

Algunos investigadores pregenerativistas intentaron establecer una escala segmental para precisar la formación de sílaba de acuerdo a las cualidades articulatorias y acústicas de los sonidos. Para De Saussure ${ }^{7}$, los sonidos se clasifican de acuerdo a su articulación basada en una abertura que va de mínima a máxima: oclusivas, fricativas, nasales, líquidas, semivocales y vocales $(i, u, u ̈)$, vocales $(a, o, o ̈)$, vocal (a). Igual clasificación fue adoptada por Sievers y Grammont $^{8}$. Jespersen ${ }^{9}$ en cambio adoptó una escala consonántica de acuerdo a la composición acústica de los sonidos que va de sonoridad mínima a sonoridad máxima: oclusivas sordas, oclusivas sonoras, fricativas sonoras, nasales y laterales, $r$, vocales altas $(i, u, \ddot{u})$, vocales medias $(e, o, o ̈)$, vocales bajas $(a, \mathfrak{x})$.

Trabajos recientes de fonología generativa natural 10 han incorporado estas clasificaciones para encontrar una explicación en términos de

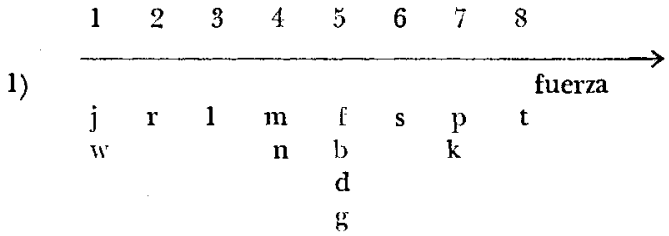

5 JOAN B. HOOPER, An introduction to natural generative phonology, Academic Pres, 1976. Esta obra es una versión revisada de su tesis doctoral mimeografiada y distribuida por Indiana University Linguistic Club en 1974 bajo el título de Aspects of natural generative phonology.

6 KathleEn Houlihan, The role of word boundary in phonological processes, tesis, University of Texas at Austin, 1975, pp. 90-111.

7 Curso de lingüistica general, Buenos Aires, 1967.

8 Véase Boshlav HÁla, La silaba: su naturaleza, su origen y sus transformaciones, trad. E. R. Palavecina y A. Quilis, Madrid, 1966.

9 La escala de Jespersen corresponde a la que presenta Hála en su obra citada.

10 Véase en particular Theodore Vennemann, "On the theory of syllabic phonology", Lingua, 39 (1972), 301-332 y el trabajo antes citado de Hooper. 
silabificación de los procesos fonológicos de varias lenguas. Así, Vennemann (p. 6) propuso para el islandés reglas de silabificación teniendo en cuenta una escala de fuerza para las consonantes:

Hooper (op. cit., p. 206) retoma luego dicha escala adaptándola y extendiéndola de la manera siguiente:

2)

$\begin{array}{lcccc}\begin{array}{c}\text { diptongos } \\ \text { parciales }\end{array} \text { líquidas } & \text { nasales } & \begin{array}{c}\text { continua11 } \\ \text { sonora }\end{array} & \begin{array}{c}\text { continua sorda } \\ \text { oclusiva sonora }\end{array} & \begin{array}{c}\text { oclusiva } \\ \text { sorda }\end{array} \\ \text { fuerza }\end{array}$

Para poder determinar esta jerarquía, Hooper se valió originalmen. te de cuatro tipos de evidencias provenientes de: la fonética acústica, la fonética motriz, la fonología diacrónica y la fonología sincrónica. La prueba acústica se desprende de los trabajos de Jakobson y Halle ${ }^{12}$ quienes tratan los fonones de consonantes y vocales con respecto al grado de sonoridad que éstas poseen; la vocal óptima, dicen, es la que produce mayor cantidad de energía, y la consonante óptima es la que produce una cantidad mínima de energía; CV es el tipo de sílaba óptimo. Según interpreta Hooper (op. cit., 1974, p. 92), dependiendo entonces de la reducción de energia, las consonantes se ordenan así:

3)

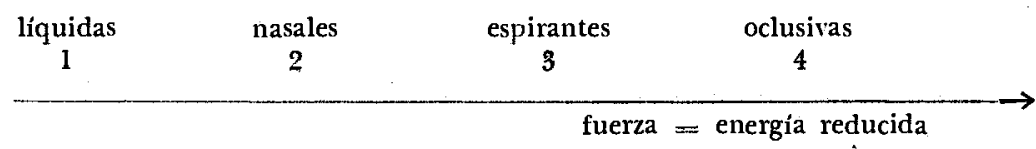

La evidencia de la fonética motriz se basa en la fuerza de articulación de las consonantes. Maléeot ${ }^{13}$ define dicha fuerza como la cantidad de energía muscular que se requiere para pronunciar las consonantes. La jerarquía de las consonantes que damos en (4) se obtuvo de un experimento hecho por Malécot donde se le preguntó a los sujetos qué sonidos les eran más difíciles de articular. La jerarquía se extiende desde las consonantes más suaves hasta las más difíciles:

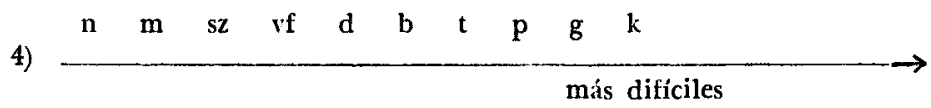

En otro experimento hecho por Malécot se trató de medir con instrumentos la presión intraoral que se necesita para la producción de las susodichas consonantes. Los resultados coincidieron en gran parte con la jerarquía (4) a pesar de que hubo una aparente contradicción, pues algunas oclusivas resultaron tener menos presión bucal que las fricativas.

Para la prueba diacrónica Hooper, que utilizó datos de Foley ${ }^{\mathbf{1 4}}$, exa-

11 En este artículo se emplean indistintamente los términos continuo, espirante y fricativa.

12 Fundamentals of Language, The Hague, 1956.

13 ANDRe MALtcot, "An experimental study of force of articulation", StL, 9 (1955), 35.44, citado por Hooper, op. cit., 1974; no se menciona en la versión de 1976.

14 Uno de los artículos en cuestión es "Systematic morphophonology", mimeogra fiado y $\sin$ fecha. Véase también James Foley, "Phonological distinctive features", FLing, 4 (1970), 87-92. 
mina algunos cambios históricos de varias lenguas y los compara con una jerarquía de fuerza. En español, por ejemplo, al realizarse el cambio consonántico proveniente del latín, las consonantes dobles, o sea las más fuertes, se redujeron a una, bucea $>$ boca; las sencillas sordas intervocálicas se convirtieron en sonoras, vita $>$ vida, las sonoras pasaron a continuas, habere $>$ haßer, y una que otra continua se perdió rode$r e>$ roer. Foley considera que el cambio $\mathrm{kk} \rightarrow \mathrm{k} \mathrm{t} \rightarrow \mathrm{d}$ y b $\rightarrow \beta$, se basa en un parentesco fonológico de estas consonantes que tienen como parámetro referencial un sistema teórico de fuerza. De modo que en un esquema de fuerza de este tipo

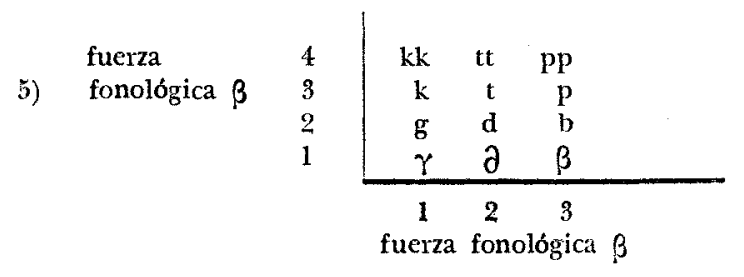

un cambio de $k k$ a $k$ implica, por ejemplo, una reducción de fuerza: una fuerza $\beta 4$ que mude a una fuerza $\beta 3$ se produce por debilitamiento ${ }^{15}$. Por lo tanto, los ejemplos antes mencionados son todos de debilitamiento.

Por último, Vennemann (art. cit., p. 6) presenta la prueba sincrónica con los procesos de asimilación que se registran en islandés. Él sugiere que según el grado en que una consonante experimente el proceso de asimilación, dicha consonante tendrá, en consecuencia, un debilitamiento relativo. En islandés la $r$ es mucho más débil que la $l, m$ y $n$ porque frente a consonantes sordas aquella se ensordese. La $s$ es más fuerte que otras espirantes porque en contextos de consonante sonora no se convierte en sonora, mientras que las otras espirantes se asimilan.

Tres de estas pruebas han sido puestas en tela de juicio; la motriz, ya dijimos, presenta una aparente contradicción. Si equiparamos, por ejemplo, dificultad de pronunciación con tensión de consonantes, los experimentos de Malécot demostraron que no todas las obstruyentes sordas fueron consideradas más difíciles que las obstruyentes sonoras y, además, las consonantes nasales y fricativas anteriores fueron consideradas más difíciles que sus respectivas posteriores. Ahora bien, de comparar la presión intraoral con la tensión de consonantes, la contradicción se agudiza, pues algunas oclusivas resultaron tener menos presión que las fricativas. Entonces, estos dos experimentos, dice Houlihan (op. cit., p. 97). ". . contradict themselves as well as contradicting the... hierarchy when their results are taken to be indication of consonantal strength".

Con la diacronía, Houlihan no está de acuerdo con el empleo del cambio que se registra en una lengua dada como fundamento para determinar una jerarquía de fuerza. En esencia, Houlihan se opone a la circularidad que mantiene Hooper al denominar "debilitamiento" a ciertos procesos para luego emplearlos como determinantes de una jerar- 
quia de fuerza y utilizar esa misma jerarquía de fuerza para determinar si un proceso es o no de debilitamiento. Hooper, por ejemplo, siguiendo muy de cerca el análisis propuesto por Foley para los cambios históricos de las vocales del alemán y del español emplea el proceso que produjo en español el cambio de vocales altas a vocales medias como prueba de que las vocales medias son en ralidad más débiles que las altas. A esto Houlihan responde: "In addition to using the same type of change to support opposite claims of strength and weakness, both Foley and Hooper seem to be claiming that if a given change $\mathrm{X}$ to $\mathrm{Y}$ is a weakening, then $\mathrm{Y}$ is weaker than $\mathrm{X}$. This type of argument cannot be used for our purposes, however, since we are considering examples of various changes and trying to determine, by comparison to some independent stregth scale, whether the change in question is a weakening or not. Therefore, we cannot assert that one change in one language is a weakening process and that the opposite change in another language is also a weakening process as the basis for claiming that the two languages have opposite strength hierarchies" (op. cit., p. 99). En seguida agrega: "We need either some independent basis for a universal strength scale, ... or some independent basis within each language for determining language specific strength and weakness" (ibid.).

Houlihan también cuestiona la prueba sincrónica pues, una vez más, Hooper emplea el proceso de debilitamiento como base para determinar una jerarquía de fuerza ya que, aparentemente, las reglas de asimilación suministran evidencias de la fuerza relativa de las consonantes. Por ejemplo, de darse la asimilación de consonantes en un contexto particular, la asimilación, dice Hooper, es producto de ese contexto, el cual debe considerarse como débil. Si, en cambio, la asimilación se da o no en un contexto, la diferencia de fuerza corresponde más bien a la consonante y no al contexto ${ }^{16}$. En vista de lo que parece ser un razonamiento circular, Houlihan afirma: "The problem with this type of evidence for our purposes in this study is that again we are asserting that certain processes are weakening processes, using that claim to determine a strenght hierarchy, and then using that strenght hierarchy to determine whether certain processes are strengthening or weakening processes" (op. cit., p. 100). De las cuatro clases de pruebas que hemos presentado, la acústica parece ser la más indicada para establecer una jerarquía universal de fuerza.

Hemos comprobado que en el español de Santo Domingo existen tres procesos en final de sílaba: 1) la fricativa $s$ que se realiza como $h$; 2) la nasal alveolar $n$ que cambia a velar y y 3) la vibrante simple $r$ que se convierte en $l$. Tomemos entonces, de modo preliminar, la versión segmental, algo modificada aquí, de la jerarquía de fuerza que propone Hooper (op. cit., p. 208) para el español hispanoamericano, que está basada en la jerarquía (2). Trataremos de ver las generalizaciones que se pueden formular: 


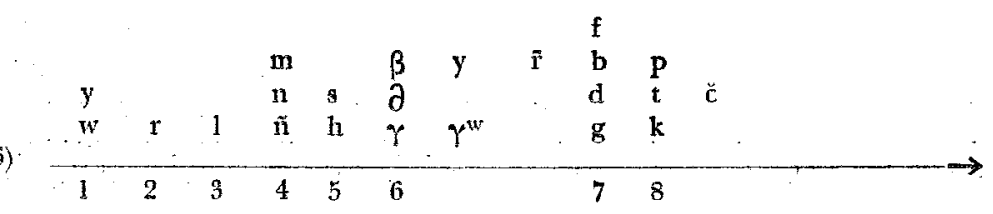

El estado de fuerza de $/ \mathrm{s} /$ frente a $/ \mathrm{h} /$ es difícil de determinar siempre y cuando nos remitamos a la escala y razonamiento de Hooper. La $s$ se muestra como segmento débil, explica ella, sólo con respecto a sus propiedades de distribución (p. 217). Es decir que para el español general, la $s$ se puede agrupar al inicio de sílaba en la medida en que puedan hacerlo las nasales y las líquidas. Una combinación de $s$ más consonante al inicio de sílaba no es posible en español. Dicha fricativa, goza, sin embargo, de amplia estabilidad en final de sílaba pues, según Hooper, posee una fuerza que se ajusta a esa posición, que en sí es de naturaleza débil. Más adelante agrega que, si en otros dialectos se convierte en aspirada, se debe precisamente a la posición final, puesto que allí todas las obstruyentes o se debilitan o desaparecen.

Su exposición circular se hace evidente: por un lado sostiene Hooper que los segmentos fuertes desaparecen en posición final porque son demasiado fuertes para dicha posición, y, por otro, afirma que un segmento se debilita o elide por ser más débil que aquellos que no sufren ninguna alteración. La comparación que hace Hooper de $s$ ante las demás obstruyentes nos pone ante la alternativa de tomar el proceso fonológico para determinar la escala, y, si esto no es factible, partir de la escala para determinar el proceso, solución circular que no conduce a ninguna parte. Hooper da, además, una explicación similar para $/ x /$, que en nuestro dialecto reemplazamos por $/ \mathrm{h} /$. El resultado final de su análisis se muestra indirectamente en la escala (6) : $s$ resulta ser más fuerte que $h$.

El comportamiento distribucional de /s/ no nos sirve como recurso para establecer su fuerza frente a $/ \mathrm{h} /$, porque se sabe que el grado de fuerza de aquélla varía considerablemente de lengua a lengua según sus posibilidades combinatorias. Se nota, sin embargo, que $s$ normalmente se modifica en $h$ como lo prueban el dialecto que aquí consideramos y lenguas como el sánscrito donde opera un proceso parecido. Si nos referimos al criterio acústico de reducción de energía = consonante óptima, quizá lo podemos tomar como base independiente para ordenar la fuerza relativa de $s$ frente a $h$. En efecto, de las consonantes fricativas, que en general se caracterizan por una ausencia bien definida de la estructura de los formantes, la óptima será aquella que muestre una tendencia hacia el sonido blanco' ${ }^{17}$. Como $h$ carece de esta tendencia, se le puede asignar un estado menos óptimo frente a $s$. Acústicamente, pues, la fricativa estridente $s$ es más fuerte que la fricativa aspirada estridente $h$.

La nasal velar y presenta un problema un tanto delicado, si es que la determinamos en base a la jerarquía. Hooper dice que, en realidad, 
no se le puede asignar un valor de fuerza pues sólo resulta de la "neutralización", en español general, ante consonante velar, y lo confirma el hecho de que nunca aparece a principio de sílaba y, por tanto, su carácter no es fonemático. De haber una nasal no anterior al inicio de sílaba, Hooper sostiene que tendrá que ser la palatal $\tilde{n}$. Aunque éste es un hecho del español en general, vimos, sin embargo, que la velar $\mathfrak{y}$ también aparece en contextos sin estar seguida de consonante velar. Recuérdese, por ejemplo, que aparece al final de palabra seguida de consonante no velar y en posición final absoluta como en sin tọ del estilo lento. La velar y, aunque no posee valor fonemático, real y efectivamente aparece como un segmento fonético del español dominicano. Se debe, por tanto, caracterizar el hecho de que en posición final de palabra aparece la velar y y no otra variante nasal; no aparece, por ejemplo, la bilabial $m$ o la dental $n$.

Jakobson y Halle nos ayudan a resolver el problema. Ellos aseguran que las consonantes difusas o anteriores - por ejemplo las nasales $m$ y $n$ - son óptimas y se distinguen de las compactas o posteriores $-\tilde{n}$ y $\mathfrak{n}$ son no óptimas- porque aquéllas poseen una gran reducción de energía acústica en la región central del espectro (op. cit., pp. 29, 42). En otras palabras, las consonantes anteriores son fuertes mientras que las posteriores son débiles. Si suponemos que la escala (2) es universal, entonces no podemos establecer por medio de ella la distinción entre consonantes anteriores y posteriores, ya que ambos fonones están agrupados arbitrariamente bajo una misma denominación fonónica: nasales. En esta escala, el orden de fuerza de $n$ con relación a y quedaría indeterminado.

Por otra parte, en la escala (6), característica del español, podríamos determinar esta relación de fuerza sólo si en la tensión 4 de las nasales el orden descendente significa aumento de energía acústica, en cuyo caso se podría añadir la $n$ inmediatamente debajo de $\tilde{n}$. Esta es una posibilidad interpretativa que hay que descartar, pues Hooper las ordena de acuerdo al grado de dificultad al pronunciarse, factor que se opone proporcionalmente al orden de Jakobson y Halle: a mayor fuerza de articulación menor será la energía para producir consonantes ${ }^{18}$. No obstante, vimos que la escala entra en conflicto directo con los resultados de los estudios hechos por Malécot y tan sólo nos resta basarnos en la acústica para obtener un orden jerárquico efectivo de las consonantes.

Para ello, Houlihan (op. cit., p. 95) propone una revisión de la escala (2) para las consonantes resonantes en la que se explícita la distinción que no hicieron Jakobson y Halle en lo que se refiere a la sonoridad de consonantes, y donde, además, se advierte que las consonantes anteriores son más fuertes que las posteriores:

7) $\begin{array}{lll}\begin{array}{lll}\text { sonora } & \text { anterior } & \text { explosión } \\ \hline \text { diptongos parciales } & \text { líquldas } & \text { nasales } \\ \hline \text { sorda } & \text { posterior } & \text { implosión }\end{array} \longrightarrow\end{array}$

18 HOOPER, 1974, p. 78. 
En esta escala bidimensional se entiende que hay un aumento de fuerza yendo de izquierda a derecha en la línea central. Además, indica que un segmento es más fuerte que el otro si contiene los fonos de la línea superior. Por ejemplo, la alveolar $n$ se caracteriza por el fonón [+ anterior] mientras que $\mathfrak{y}$ es [- anterior] y por lo tanto $\mathrm{y}$ es más débil y el proceso que cambie $n$ a $\mathfrak{y}$ puede tomar el nombre genérico de debilitamiento.

Por la escala (2) de Hooper y la (7) de Houlihan no podemos rastrear la distinción de fuerza entre $r$ y $l$ ni en el eje vertical ni en el eje horizontal. En cambio, la escala (6), presenta una jerarquización de $l$ con tensión de 3 frente a $r$ con tensión de 2 , lo cual significa que $l$ es más fuerte que $r$. La justificación de este orden que Hooper suministra se basa en la distribución de ambas en varias lenguas. Dice Hooper que para el español, la secuencia de $/ 1 \$ r /$ (el símbolo \$ representa el contorno silábico) como en /al\$rededor/ exige que la $/ r /$ se convierta en $/ \overline{\mathrm{r}} /$, mientras que lo opuesto, $/ \mathrm{r} \$ 1 /$, como en $/$ per $\$ 1 \mathrm{l} /$, la $/ 1 /$ permanece inmodificada ${ }^{10}$. El cambio de tensión para la $/ \mathrm{r} /$ inicial -dice Hooperse puede formular en base al principio de que la primera consonante de una sílaba es más fuerte que la consonante final de la sílaba precedente. Dice, además, que la diferencia de fuerza entre $t$ y $d$ y la fuerza de $l$ no es suficiente para permitir que $t, d$ y $l$ formen grupos consonánticos. Por eso se explica que hayan en español los grupos $t r$ y $d r$ y no los grupos*tl y*dl (ibid., p. 212).

Si hemos de aceptar que $l$ es más fuerte que $r$ en razón de su incapacidad combinatoria con obstruyentes débiles, los hechos del español no sostienen estas premisas. Las consonantes velares $k$ y $g$ al ser posteriores, según dijimos, se caracterizan por su relativa debilidad; por consiguiente, debería excluirse la existencia de los grupos consonánticos $k l$ y $g l$. Numerosos ejemplos, y para citar unos cuantos, clara, clima, glándula, glucosa, muestran estas velares seguidas de $l$ como posibles y factibles combinaciones del español. El que $l$ no pueda agruparse con $t$ o $d$ no obedece a que sea más fuerte que $r$ ni tampoco a que las dentales sean más débiles que las labiales o velares ${ }^{20}$. La pronunciación de las pala-

10 HOOPER, 1976, p. 212.

20 Según Hooper, las consonantes coronales son, al parecer, más débiles que las labiales y velares; presenta como prueba la $d$ final del sufijo dad que se hace frica-

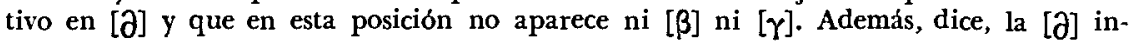
tervocálica es la que tiende a desaparecer con más frecuencia (véase p. 215). Es indudable que la [ə] de los participios verbales y de uno que otro sustantivo y adjetivo tiende a desaparecer en posición intervocálica, pero no es menos cierto que [ $\beta$ ] y $[\gamma]$ también se transforman o desaparecen. En Cuba se han registrado formas como la [wóka] por la boca, [kúwa] por Cuba, [báwa] por baba (véase Crusrina Isbasescu, "Algunas peculiaridades fonéticas del español hablado en Cuba", $R R L, 10$ (1965), p. 580. En Nuevo México, Aurelio Espinosa (Estudios sobre el español de Nuevo Méjico, Biblioteca de Dialectología Hispanoamericana, 1930, t. 1, pp. 227, 234) presenta elisión de $g$ en palabras como luego [lwéo], hago [áo], me gusta [me ústa]. $\beta$ también se elimina en formas como trabajo [traáho], no bebas [no éßas]. El proceso parece extenderse aún a la ciudad de México donde también se elimina $g$ (véase PEDro Henrígez Ureña, "Observaciones sobre el español en América", RFE, 8 (1921), p. 366: agua [áwa], aguacate [awakáte]. Todas ellas muestran que, aparentemente, 
bras atlas y Atlántico que se registra en Santo Domingo niegan estas aseveraciones. El dominicano las pronuncia respectivamente como [á\$tla (h) ] y [a\$tlántiko], división silábica que encaja perfectamente con la regla de silabificación del español, donde se estipula que, en caso de haber una obstruyente como $t$ o $d$ seguida de consonante líquida, el contorno silábico habrá de aparecer inmediatamente ante la obstruyente. Es decir que la existencia dental sorda ante líquida lateral no se puede excluir del español. Que se sepa, hasta ahora no se ha registrado el grupo consonántico inicial $d l$. Su ausencia sugiere que deben haber otros factores, y no precisamente de fuerza, que impiden su realización fonética.

Con el estado incierto de $r$ frente a $l$ se abren tres posibilidades para establecer una escala jerárquica para el español y dos de ellas serían inmotivadas. La primera sería aceptar la escala (6) como está, es decir suponer que $r$ es más débil que $l$. Recordemos, empero, que la $r$ final se convierte en $l, y$, si nos remitimos a esta jerarquía, concluiremos que el proceso es de fuerza contraria a lo que afirma Hooper, para quien los procesos finales de sílaba son de debilitamiento.

La segunda posibilidad sería modificar la jerarquía de modo que $r$ quede en una posición más elevada en el eje horizontal. El cambio de $r$ a $l$ sería entonces de debilitamiento, lo que, en consecuencia, da una aparente motivación a la jerarquía. Esta solución no nos saca del paso, puesto que en la zona suroeste del país se verifica el cambio de $l$ a $r$ en posición final según lo documenta Jiménez Sabater ${ }^{21}$. Una palabra como falda se pronuncia [fárda]. Nuevamente fallaría la hipótesis de Hooper, ya que en final de sílaba tendríamos un proceso de fuerza.

Es evidente que no podemos asignar a $r$ una mayor fuerza que a $l$ y viceversa, porque ambas experimentan procesos en direcciones opuestas. Esto nos induce a sugerir, como tercera posibilidad, asigarles a ambas líquidas fuerzas idénticas en la escala jerárquica. Al no poseer datos acústicos que las relacionen jerárquicamente, los procesos fonológicos de $r \rightarrow 1$ y $l \rightarrow r$ nos pueden servir como motivos para emparentarlas con un único valor.

las modificaciones que experimentan no tienen nada que ver con su fuerza relativa, sino que probablemente se debe a la composición fonónica del segmento, pues es posible que lo que se debilita o elide sea simplemente un fonón. Esas modificaciones también pueden resultar de la secuencia de los segmentos o quizá provengan de la posición en que se encuentran en la silaba (véase el trabajo de PETER C. BJARKMAN, "Weakening chains and the natural histories of selected Spanish consonants", ponencia presentada en The eighth annual linguistic symposium on Romance languages, ei 9-11 de marzo 1978 en la Universidad de Louisville, Kentucky). Con respecto a esta última posibilidad, debe observarse que las consonantes de los ejemplos antes citados no se hallan en posición final de sílaba sino en posición inicial, hecho que demuestra que los procesos de debilitamiento y elisión pueden efectuarse al inicio de sílaba. Nótese además que aqui no debe interpretarse inicio de sílaba por inicio de grupo fónico, aunque ambos pueden coincidir, ya que los procesos aludidos se realizan en la primera situación, pero no en la segunda. Así de agua buena se puede obtener [áwa $\beta$ wéna], perc en gato bueno no se cumple [*áto $\beta$ wéno] (Bjarkman, art. cit.).

21 MaX A. Jiméney SABater, Más datos sobre el español de la Repuiblica Dominicana, Santo Domingo, 1975. 
El cambio de $r$ a $l$ en Santo Domingo no sería ni de refuerzo ni de debilitamiento; sería, únicamente, un proceso de neutralización en final de sílaba. $\mathrm{Si}$, por el contrario, nos fijamos en la modificación de $n$ a: $\eta$ notamos un proceso que no se debe especificar como de neutralización, ya que $\eta$ no contrasta en la representación subyacente con ninguna otra nasal en posición final. Es, por tanto, un proceso de debilitamiento. Por otra parte, como ya dijimos, la conversión de $r$ a $l$ ejemplifica una neutralización porque ambas existen y contrastan en posición inicial de sílaba en interior de palabra como en / pára/-/pála/, y en posición final, como se observa en forma fonética [káldo] que proviene de /kárdo/ o /káldo/.

De acuerdo a lo dicho, no tendríamos ninguna razón para rechazar la hipótesis de Houlihan: "Word-final neutralizations may be strengthening processes. Word-final non-neutralizations are weakening processes" (op. cit., p. 111).

Con ella no se invalida y tampoco se reajusta la jerarquía de fuerza que se ha establecido en (7). Damos, por tanto, apoyo a la hipótesis de Houlihan en tanto no aparezcan en un dialecto o en una lengua reglas de no neutralización al final de sílaba que sean un proceso de fuerza.

En resumen, un proceso que englobe una modificación de fonones al final de sílaba puede ser de debilitamiento en la medida que se le compare con una jerarquía consonántica de fuerza que lo determine $\mathrm{y}$ defina. Y, finalmente, no todos los procesos finales de sílaba son necesariamente de debilitamiento.

Rafael NúÑez Cedeño

University of New Hampshire.

\section{OVIEDO A MEDIA LUZ}

Recia personalidad la de Gonzalo Fernández de Oviedo, alias de Sobrepeña. Recia y longeva personalidad la del Néstor de los cronistas de Indias, según la hermosa y acertada expresión de Eugenio Asensio. Había nacido en Madrid, y en 1477 o 1478, vacilación impuesta por el hecho de que en esto de calcular su edad, nuestro cronista no las cortaba en el aire, y no se conoce ningún testimonio documental sobre ella. Si el año exacto de su nacimiento se mantiene reacio e incierto', no ocurre lo

1 No quiero andarme por las ramas y dejar suponer que malsino la memoria de Oviedo, o que arremeto contra algún critico en particular. Un solo texto autógrafo de Oviedo basta para iluminar la problemática cuestión. En la dedicatoria al futuro Felipe II de sus Quinquagenas de la nobleza de España, nuestro cronista escribió de su mano: "A diez días del mes de Enero de 1555 años de la natalidad de Christo nuestro Redemptor, de mi propria e cassada [sic] mano, e seyendo complidos 77 años de mi edad". Esto nos debe retrotraer al año de 1478 , lo que queda refrendado de inmediato por los cómputos que hace acerca de cuándo y cuántos años tenía cuando 